\title{
SiamL.
}

\section{Computing Equilibria of N-Person Games}

Author(s): Robert Wilson

Source: SIAM Journal on Applied Mathematics, Vol. 21, No. 1 (Jul., 1971), pp. 80-87

Published by: Society for Industrial and Applied Mathematics

Stable URL: http://www.jstor.org/stable/2099844

Accessed: 09/12/2010 16:36

Your use of the JSTOR archive indicates your acceptance of JSTOR's Terms and Conditions of Use, available at http://www.jstor.org/page/info/about/policies/terms.jsp. JSTOR's Terms and Conditions of Use provides, in part, that unless you have obtained prior permission, you may not download an entire issue of a journal or multiple copies of articles, and you may use content in the JSTOR archive only for your personal, non-commercial use.

Please contact the publisher regarding any further use of this work. Publisher contact information may be obtained at http://www.jstor.org/action/showPublisher?publisherCode=siam.

Each copy of any part of a JSTOR transmission must contain the same copyright notice that appears on the screen or printed page of such transmission.

JSTOR is a not-for-profit service that helps scholars, researchers, and students discover, use, and build upon a wide range of content in a trusted digital archive. We use information technology and tools to increase productivity and facilitate new forms of scholarship. For more information about JSTOR, please contact support@jstor.org. 


\title{
COMPUTING EQUILIBRIA OF N-PERSON GAMES*
}

\author{
ROBERT WILSON†
}

\begin{abstract}
The algorithm of Lemke and Howson for finding an equilibrium of a 2-person game is extended to provide a constructive procedure for finding an equilibrium of an $N$-person game by finding in succession an equilibrium for each of certain related $k$-person games, $1 \leqq k \leqq N$.
\end{abstract}

1. Introduction. The purpose of this paper is to demonstrate that the algorithm of C. E. Lemke and J. T. Howson [1], originally formulated as a constructive procedure for finding an equilibrium of a 2-person game, can, in fact, be extended directly to construct an equilibrium of an $N$-person game. Of course, the procedure requires the solution of multilinear equations in the general case, rather than simply linear equations as in the 2-person case, but presumably there are or will be numerical methods adequate to this task. Nash [3] gives an example of a 3-person game with rational data and an irrational equilibrium.

The central idea is that, in general, an almost-complementary path leads to an equilibrium, just as in the 2-person case. Moreover, one can find a point on an almost-complementary path with which to initiate the procedure by constructing an equilibrium of an $(N-1)$-person game. Hence, one can construct an equilibrium of an $N$-person game by constructing in succession equilibria of certain $k$-person games, $1 \leqq k \leqq N-1$.

2. Formulation. We consider an $N$-person noncooperative game $\Gamma_{N}$ in normal form (cf. Nash [3] or Luce and Raiffa [2,pp. 170-173]). Let $P=\{1, \cdots, N\}$ be the finite set of players, and for $n \in P$ let $S_{n}$ be the finite set of pure strategies available to player $n$ in the normal-form description. Then $\pi=X_{n \in P} S_{n}$ is the set of possible plays of the game. For each $n \in P$ and $\omega \in \pi$ we are given $u_{\omega}^{n}$, the utility to player $n$ from the play $\omega$. We assume without loss of generality that $(n \in P)(\omega \in \pi) u_{\omega}^{n}<0$, and let $a_{\omega}^{n}=-u_{\omega}^{n}>0 .{ }^{1}$ Define the array $A^{n}=\left(a_{\omega}^{n}\right)_{\omega \in \pi}$ with $N$ attributes and positive elements $\left(A^{n}>0\right)$.

A mixed strategy for a player $n \in P$ is a probability distribution, say $\xi^{n}=\left(\xi_{i}^{n}\right)_{i \in S_{n}}$, over his pure strategies in $S_{n}$; that is, $\left(i \in S_{n}\right) \xi_{i}^{n} \geqq 0$ and $\sum_{i \in S_{n}} \xi_{i}^{n}=1$. Thus, $\xi^{n}$ is an element of the face of the $\left|S_{n}\right|$-dimensional unit simplex $\sigma_{n}$, and $\xi_{i}^{n}$ is the probability with which player $n$ uses his $i$ th pure strategy. Define $\Xi=X_{n \in P} \sigma_{n}$, the collection of mixed strategy combinations for the game. Also, for each player $v \in P$ and $\xi \in \Xi$ let $\Xi_{v}(\xi)=\left\{\xi \mid \xi \in \Xi,(n \neq v) \xi^{n}=\xi^{n}\right\}$, the subcollection with player $v$ 's mixed strategy variable. In general, for any $x \in E^{\pi}$, the Euclidean space of $K=\sum_{n \in P}\left|S_{n}\right|$ dimensions, where $x=\left(\left(x_{i}^{n}\right)_{i \in S_{n}}\right)_{n \in P}$, let

$$
\begin{aligned}
& A_{i}^{n}(x)=\sum_{\substack{\omega \in \pi \\
\omega_{n}=i}} a_{\omega}^{n}\left(\prod_{\substack{\omega_{v}=j \\
v \neq n}} x_{j}^{v}\right), \quad i \in S_{n}, \quad n \in P ; \\
& A^{n}(x)=\sum_{i \in S_{n}} A_{i}^{n}(x) x_{i}^{n}, \quad n \in P .
\end{aligned}
$$

* Received by the editors September 8, 1969, and in revised form January 23, 1970.

† Graduate School of Business, Stanford University, Stanford, California 94305. This work was supported by the Atomic Energy Commission under Contract AT(04-3)-326 PA \#18, and first appeared as Working Paper 163, Graduate School of Business, Stanford University, August 1969.

${ }^{1}$ Universal quantifiers are represented by parentheses ; e.g., $(n \in P)$ means "for all $n \in P$." 
In particular, a mixed strategy combination $\xi \in \Xi$ yields an expected utility of $-A_{i}^{n}(\xi)$ to player $n$ conditionally on his pure strategy $i$, and an unconditional expected utility of $-A^{n}(\xi)$.

An equilibrium is a mixed strategy combination $\xi \in \Xi$ for which

$$
(n \in P)\left(\bar{\xi} \in \Xi_{n}(\xi)\right) A^{n}(\xi) \leqq A^{n}(\bar{\xi}),
$$

so that no player wishes to change his mixed strategy alone. Since $\xi^{n}$ could assign probability one to any one of $n$ 's pure strategies, it is readily verified that an equivalent definition is that $(n \in P)\left(i \in S_{n}\right) A_{i}^{n}(\xi) \geqq A^{n}(\xi)$. Moreover, one can eliminate the restriction $\xi \in \Xi$ by transforming variables as follows: Define

$$
\begin{aligned}
& \alpha_{n}=\left[\frac{\prod_{v \neq n} A^{v}(\xi)}{A^{n}(\xi)^{N-2}}\right]^{1 /(N-1)}, \\
& x^{n}=\alpha_{n}^{-1} \xi^{n}, \quad n \in P ;
\end{aligned}
$$

then, for an equilibrium it is necessary and sufficient to find $x, y \in E^{\pi}$ for which

$$
(n \in P)\left(i \in S_{n}\right) x_{i}^{n} \geqq 0, \quad y_{i}^{n} \geqq 0, \quad A_{i}^{n}(x)-y_{i}^{n}=1, \quad x_{i}^{n} y_{i}^{n}=0 .
$$

The explicit derivation of (3) parallels the exposition of Lemke and Howson for the 2-person case. Ordinarily, if we are given $x \in E^{\pi}$ we assume $y$ to be defined implicitly by $y_{i}^{n}=A_{i}^{n}(x)-1$.

From a solution $(x, y)$ to $(3)$ one obtains an equilibrium $\xi \in \Xi$ via the reverse transformation $(n \in P) \xi^{n}=\left(\sum_{i \in S_{n}} x_{i}^{n}\right)^{-1} x^{n}$. Consequently, the problem of constructing a solution to (3) is the immediate subject of our investigation.

3. Geometric characterization. For each player $n \in P$ let

$$
X_{n}=\left\{x \mid x \in E^{\pi}, x \geqq 0,\left(i \in S_{n}\right) A_{i}^{n}(x) \geqq 1\right\}
$$

and let $Z^{*}=\cap_{n \in P} X_{n}$. Clearly each $X_{n}$ is closed and nonempty, since $A^{n}>0$, and each $X_{n}$ and $Z^{*}$ contain every solution to (3). Nash [3] has given a fixedpoint proof of the existence of an equilibrium, so $Z^{*}$ is nonempty, although later we shall provide a constructive proof of this fact.

It will suffice here to consider a pertinent subset of $Z^{*}$, namely, the set $Z$ of points from $Z^{*}$ for which $x_{i}^{n}\left(A_{i}^{n}(x)-1\right)>0$ for at most one pair $(n, i), n \in P$, $i \in S_{n}$. Such a point is said to be almost complementary, or $(n, i)$-almost-complementary, and if $x$ is $(n, i)$-almost-complementary but $x_{i}^{n}\left(A_{i}^{n}(x)-1\right)=0$ then $x$ is said to be complementary. Clearly, $Z$ contains all solutions to (3), and a point $x \in E^{\pi}$ solves (3) if and only if $x$ is a complementary point in $Z$.

A point in $E^{\pi}$ is a vector of dimension $K=\sum_{n \in P}\left|S_{n}\right|$. On the other hand, a point in $Z \subset E^{\pi}$ is almost complementary, which requires that it satisfy at least $K-1$ equalities of the form $x_{i}^{n}=0$ or $A_{i}^{n}(x)=1$, called the boundary conditions of $Z^{*}$. Hence, one expects that ordinarily $Z$ will be a graph of dimen- 
sionality $K-(K-1)=1$. But to ensure this we impose the following restriction throughout. ${ }^{2,3}$

Nondegeneracy assumption: No point in the nonnegative orthant of $E^{\pi}$ satisfies more than $K$ boundary conditions, and no two points in the nonnegative orthant of $E^{\pi}$ satisfy the same $K$ boundary conditions.

With this convention, $Z$ is a unidimensional graph in $E^{\pi}$ and it is worthwhile to distinguish its nodes and arcs.

A node of $Z$ is a point in $Z$ that is an extreme point of $Z^{*}$; that is, it satisfies (precisely) $K$ boundary conditions. Clearly, there is only a finite number of nodes of $Z$, and a point $x \in E^{\pi}$ solves (3) if and only if it is a complementary node in $Z$.

Similarly, an $\operatorname{arc}$ of $Z$, corresponding to an open edge of $Z^{*}$, is the collection of points in $Z$ satisfying some specified set of (precisely) $K-1$ boundary conditions as one varies parametrically some value $x_{i}^{n}$ or $y_{i}^{n}$ in the open interval from zero, at an extreme point of $Z^{*}$, to the first value at which another extreme point (if any) is encountered. Clearly, an arc "leaves" $Z^{*}$ only at an extreme point of $Z^{*}$; that is, at a node of $Z$. Note that for $N \geqq 3$ an arc is nonlinear; actually, multilinear.

The idea of a constructive procedure, due originally to Lemke and Howson, is to proceed from one almost-complementary node to another, along the almostcomplementary arcs, until a complementary node is found, which must solve (3) and yield an equilibrium.

4. Almost-complementary paths. For some fixed player $m \in P$ and a pure strategy choice $j \in S_{m}$, let $Z(m, j)$ be the subset of $(m, j)$-almost-complementary points in $Z$. Clearly, for each pair $(m, j), x$ solves (3) if and only if $x$ is a complementary node in $Z(m, j)$, so the choice of $(m, j)$ may be arbitrary.

Lemma 1. A node in $Z(m, j)$ has either one or two $(m, j)$-almost-complementary arcs starting from it, and there is just one if and only if the node is complementary.

Proof. A node $x \in Z(m, j)$ satisfies precisely $K$ boundary conditions and is either complementary or almost complementary. If $x$ is complementary, then precisely one of each pair $\left(x_{i}^{n}, y_{i}^{n}\right)$ is zero, and only that arc starting from $x$ that is parametrized by the one of $x_{j}^{m}$ or $y_{j}^{m}$ that is zero is $(m, j)$-almost-complementary. Otherwise, $x$ is not complementary but almost complementary with both $x_{j}^{m}$ and $y_{j}^{m}$ positive, and there is a unique pair $(n, i)$ for which both $x_{i}^{n}$ and $y_{i}^{n}$ are zero. In this case, only the two arcs starting from $x$ that are parametrized by $x_{i}^{n}$ and $y_{i}^{n}$ respectively are $(m, j)$-almost-complementary, and these are distinct. This concludes the proof.

Similar arguments show that a complementary node in $Z$ has precisely $K$ arcs starting from it, whereas a node that is not complementary has precisely two and belongs to just one $Z(m, j)$. A maximal connected set of $(m, j)$-almostcomplementary nodes and arcs is called an $(m, j)$-path. Clearly, $Z(m, j)$ is the union of a finite number of $(m, j)$-paths.

\footnotetext{
${ }^{2}$ Lemke and Howson [1] give a procedure for perturbing a degenerate problem to satisfy this assumption, but we shall not consider such extensions here.

${ }^{3}$ This is sufficient to ensure the nondegeneracy of all of the derived games $\Gamma_{k}, 1 \leqq k \leqq N$, encountered later in Theorem 1 and $\S 5$.
} 
Let $\Gamma_{N-1}(m, j)$ be the $(N-1)$-person game among the other players that results from assuming that player $m$ uses his strategy $j$ with probability one (i.e., $\left.\xi_{j}^{m}=1,\left(i \in S_{m}, i \neq j\right) \xi_{i}^{m}=0\right)$.

Lemma 2. An equilibrium of $\Gamma_{N-1}(m, j)$ corresponds to an $(m, j)$-almost-complementary node in $Z(m, j)$.

Proof. Let $\left(\xi^{n}\right)_{n \neq m}$ be an equilibrium of $\Gamma_{N-1}(m, j)$. We distinguish two cases : either $N \geqq 3$ or $N=2$. If $N \geqq 3$, then let the transformation (2) of $\left(\xi^{n}\right)_{n \neq m}$ in the $(N-1)$-person game $\Gamma_{N-1}(m, j)$ be $\left(\bar{x}^{n}\right)_{n \neq m}$, so that $\left(\bar{x}^{n}\right)_{n \neq m}$ satisfies (3) for $\Gamma_{N-1}(m, j)$. This can be converted to the $N$-person game $\Gamma_{N}$ by defining $\left(i \in S_{m}\right)\left[\bar{x}_{i}^{m}=1\right.$ if $i=j$, and $\bar{x}_{i}^{m}=0$ if $\left.i \neq j\right]$. Then $\bar{x}=\left(\bar{x}^{n}\right)_{n \in P}$ satisfies (3) for $\Gamma_{N}$ except possibly for $n=m$. Define $\beta=\min _{i \in S_{m}} A_{i}^{m}(\bar{x})$ and let $i^{*} \in S_{m}$ yield the minimum. Then define

$$
\begin{aligned}
(n \in P, n \neq m) x^{n} & =\beta^{-1 /(N-1)} \bar{x}^{n}, \\
x^{m} & =\beta^{(N-2) /(N-1)} \bar{x}^{m} .
\end{aligned}
$$

Letting $x=\left(x^{n}\right)_{n \in P}$ one obtains

$$
\begin{aligned}
(n \in P, n \neq m)\left(i \in S_{n}\right) A_{i}^{n}(x) & =A_{i}^{n}(\bar{x}) x_{j}^{m}\left[\beta^{-1 /(N-1)}\right]^{N-2}=A_{i}^{n}(\bar{x}), \\
\left(i \in S_{m}\right) A_{i}^{m}(x) & =A_{i}^{m}(\bar{x})\left[\beta^{-1 /(N-1)}\right]^{N-1} \\
& =A_{i}^{m}(\bar{x}) / \beta \\
& =A_{i}^{m}(\bar{x}) / A_{i^{*}}^{m}(\bar{x}) \geqq 1 \quad\left(\text { with }=1 \text { if } i=i^{*}\right) .
\end{aligned}
$$

Hence, $x$ satisfies (3) excepting only the possibility that $x_{j}^{m} y_{j}^{m}>0$, so $x$ is $(m, j)$ almost-complementary, $x \in Z(m, j)$, and also $x$ is a node. The proof if $N=2$, which is implicit in Lemke and Howson's exposition, is rather different. For $n \neq m$ the fact that $\xi^{n}$ is an equilibrium requires $\left(i \in S_{n}\right)\left[\xi_{i}^{n}=1\right.$ if $i=\omega_{n}^{*}$, and $\xi_{i}^{n}=0$ if $\left.i \neq \omega_{n}^{*}\right]$, where $\omega^{*} \in \pi$ yields $\min _{\omega_{m}=j} a_{\omega}^{n}$. Let $\omega^{* *}$ yield $\min _{\omega_{n}=\omega_{n}^{*}} a_{\omega}^{m}$ and define

$$
\begin{aligned}
& \left(i \in S_{m}\right)\left[x_{i}^{m}=1 / a_{\omega^{*}}^{n} \text { if } i=j, \quad \text { and } \quad x_{i}^{m}=0 \text { if } i \neq j\right] \\
& \left(i \in S_{n}\right)\left[x_{i}^{n}=1 / a_{\omega^{* *}}^{m} \text { if } i=\omega_{n}^{*}, \quad \text { and } \quad x_{i}^{n}=0 \text { if } i \neq \omega_{n}^{*}\right]
\end{aligned}
$$

Let $x=\left(\left(x_{i}^{n}\right)_{i \in S_{n}}\right)_{n \in P}$. Then one finds :

$$
\begin{gathered}
\left(i \in S_{m}\right) A_{i}^{m}(x)=a_{\left(i, \omega_{n}^{*}\right)}^{m} x_{\omega_{n}^{*}}^{n}=a_{\left(i, \omega_{n}^{*}\right)}^{m} / a_{\omega^{* *}}^{m} \geqq 1 \quad\left(\text { with }=1 \text { if } i=\omega_{m}^{* *}\right), \\
\left(i \in S_{n}\right) A_{i}^{n}(x)=a_{(j, i)}^{n} x_{j}^{m}=a_{(j, i)}^{n} / a_{\omega^{*}}^{n} \geqq 1 \quad\left(\text { with }=1 \text { if } i=\omega_{n}^{*}\right) .
\end{gathered}
$$

Hence, as before, $x$ is $(m, j)$-almost-complementary, $x \in Z(m, j)$, and $x$ is a node. This concludes the proof of the lemma.

A node of $Z(m, j)$ that arises from an equilibrium of $\Gamma_{N-1}(m, j)$ is called an initial node of $Z(m, j)$ and is identified by the feature that $x_{i}^{m}$ is positive only for $i=j$. The initial nodes are the endpoints of the unbounded arcs of $Z(m, j)$, as we shall now establish.

LEMMA 3. An arc in $Z(m, j)$ that starts from any node other than an initial node is bounded, and an initial node is the endpoint of precisely one unbounded arc.

Proof. The proof of Lemma 3 is broken up into several simpler propositions. 
Let $x^{*}$ be a node in $Z(m, j)$ which is not an initial node of $Z(m, j)$, and let $T$ be an arc of $Z(m, j)$ that starts from $x^{*}$.

Proposition 1. For each $n \in P$ there exists $i(n) \in S_{n}$ for which $(x \in T) A_{i(n)}^{n}(x)=1$.

Proof. Suppose to the contrary that (since $T$ is an arc) for some $n \in P, x \in T$, $\left(i \in S_{n}\right) A_{i}^{n}(x)>1$. Then for $x$ to be $(m, j)$-almost-complementary requires that either (a) $x^{n}=0$ if $n \neq m$, or (b) $\left(i \in S_{m}, i \neq j\right) x_{i}^{m}=0$ if $n=m$. In case (a), for any $v \in P$, $v \neq n, i \in S_{v}$, one has $A_{i}^{v}(x)=0<1$, contradicting $x \in Z(m, j) \subset X_{v}$. In case (b), the same property must hold for $x^{*}$, contradicting the assumption that $x^{*}$ is not an initial node of $Z(m, j)$.

Proposition 2. If for some $n \in P, i \in S_{n}, x_{i}^{n}$ is unbounded along $T$, then $\left(i \in S_{v}\right) x_{i}^{v} \rightarrow 0$ along $T$ for some $v \in P$.

Proof. Define $A_{i j}^{n v}(x)$ in the obvious way so that $A_{i}^{n}(x)=\sum_{j \in S_{v}} A_{i j}^{n v}(x) x_{j}^{v}$, and similarly define $A_{i j k}^{n v_{1} v_{2}}(x)$, etc. Suppose, say, that $x_{1}^{1}$ is unbounded along $T$ but, contrary to the proposition, for each $v \in P$ there is some $j(v) \in S_{v}$ for which it is false that $x_{j(v)}^{v} \rightarrow 0$ along $T$. By letting $i(2)=1$ for definiteness, the condition along $T$ that $1=A_{1}^{2}(x)=\sum_{i \in S_{1}} A_{1 i}^{21}(x) x_{i}^{1}$ requires that $A_{11}^{21}(x) \rightarrow 0$ along $T$. If there were only two players, then $A_{11}^{21}(x)=a_{11}^{2}$, a contradiction, so there is a third player for which $A_{11}^{21}(x)=\sum_{j \in S_{3}} A_{11 j}^{213}(x) x_{j}^{3} \rightarrow 0$ along $T$. If $j(3)=1$, then this requires $A_{111}^{213}(x) \rightarrow 0$ along $T$. Hence there is a fourth player, etc., and one can repeat the process until the set of players is exhausted and one has found a contradiction.

Proposition 3. If $T$ is unbounded, then there are at least three players (i.e., $N \geqq 3$ ), and $x^{v} \rightarrow 0$ along $T$ for at least two players $v \in P$.

Proof. The proof of Proposition 2 actually shows that if some $x_{i}^{n}$ is unbounded along $T$, then for each $\vec{n} \neq n$, the condition $A_{i(\bar{n})}^{\bar{n}}(x)=1$ implies the existence of a $v \in P, v \neq \bar{n}, v \neq n$, for which $x^{v} \rightarrow 0$. Hence, there are at least three players. Moreover, by choosing $\bar{n}=v$ and repeating the construction one must obtain a second player $v^{\prime}$ for which $x^{v^{\prime}} \rightarrow 0$.

Proposition 4. $T$ is bounded.

Proof. For each $x \in E^{\pi}, \omega \in \pi$, and $n \in P$, define $h_{n}(x, \omega)=\prod x_{j}^{v}$, where the product is over those $(v, j)$ for which $\omega_{v}=j$ and $v \neq n$. Thus, $A_{i}^{n}(x)=\sum a_{\omega}^{n} h_{n}(x, \omega)$, where the sum is over those $\omega \in \pi$ for which $\omega_{n}=i$. According to Proposition 1, $(n \in P)(x \in T) A_{i(n)}^{n}(x)=1$. Consequently, there exists a positive constant $b$ such that $(n \in P)(\omega \in \pi)(x \in T) h_{n}(x, \omega)<b$, and for each $n \in P$ there exists an $\omega(n) \in \pi$ for which it is false that $h_{n}(x, \omega(n)) \rightarrow 0$ along $T$. Now, suppose, contrary to the proposition, that say $x_{1}^{1}$ is unbounded along $T$. Choose that $\bar{\omega} \in \pi$ for which $\bar{\omega}_{1}=1$ and $(n \in P, n \neq 1) \bar{\omega}_{n}=\omega_{n}(1)$; clearly, $(x \in T) h_{1}(x, \bar{\omega})=h_{1}(x, \omega(1))$ and therefore it is false that $h_{1}(x, \bar{\omega}) \rightarrow 0$ along $T$. Define $H_{n}(x, \omega)=\prod h_{v}(x, \omega)$, where the product is over $v \in P, v \neq n$. Clearly, $H_{n}(x, \omega)<b^{N-1}$ along $T$. Observe, however, that $H_{1}(x, \bar{\omega})=\left(x_{1}^{1}\right)^{N-1} h_{1}(x, \bar{\omega})^{N-2}$. Hence, since $x_{1}^{1}$ is unbounded along $T$ by supposition, and Proposition 3 assures that $N \geqq 3, h_{1}(x, \bar{\omega}) \rightarrow 0$ along $T$. This is a contradiction, and the proof that $T$ is bounded is complete.

The same reasoning shows that if $x^{*}$ is an initial node of $Z(m, j)$, but $x^{*}$ is not complementary and $x_{i}^{* m}=A_{i}^{m}\left(x^{*}\right)-1=0$ for some $i \in S_{m}, i \neq j$, then the $(m, j)$-almost-complementary arc $T$ that is parametrized by $x_{i}^{m}$ must be bounded, since Proposition 1 is again valid with $i(m)=i$. Finally, whether or not $x^{*}$ is complementary, since each $A^{n}>0$ it is clear that the $(m, j)$-almost-complementary 
arc parametrized by $y_{i}^{m}$ ( $i=j$ if $x^{*}$ is complementary) is unbounded. This completes the proof of Lemma 3.

THEOREM 1. The number of equilibria is positive and odd.

Proof. The proof is by induction on the number of players. Clearly, a (nondegenerate) 1-person game has a single equilibrium. Assume therefore that a (nondegenerate) $(N-1)$-person game has an odd number of equilibria. Then $\Gamma_{N-1}(m, j)$ has an odd number of equilibria, and therefore $Z(m, j)$ has an odd number of initial nodes. Now starting from an arbitrary node $x$ in $Z(m, j)$ there are either one or two $(m, j)$-almost-complementary arcs, and just one whenever $x$ is complementary. Also one such arc is unbounded if and only if $x$ is an initial node. Traversing a bounded arc, one arrives at another node, say $x^{1}$. Now, if $x^{1}$ is complementary, then there is no exit from $x^{1}$ on a new $(m, j)$-almost-complementary arc; if $x^{1}$ is an initial node, then the only exit is along an unbounded arc; and, otherwise, there is precisely one such exit along another bounded arc. Each $(m, j)$-almost-complementary arc starting from $x$ proceeds therefore through a finite number of nodes of $Z(m, j)$ until it terminates in either a complementary node, or an unbounded arc, or returns to $x$ (to return to any other node, say $x^{1}$, would imply the existence of three $(m, j)$-almost-complementary arcs starting from $\left.x^{1}\right)$. In the latter case, $x$ is on an $(m, j)$-path that is circular and contains no complementary nodes. The two former cases resolve into whether the $(m, j)$-path containing $x$ has 0,1 , or 2 initial nodes. If there are no initial nodes, then the terminus at each of the two ends must be a complementary node. If there is one initial node and therefore one unbounded arc, then the other terminus must be a complementary node. If there are two initial nodes, then the path terminates in an unbounded arc at both ends, and consequently there is no complementary node. Now, the induction hypothesis assures that there are an odd number of initial nodes; hence, there are an odd number of complementary nodes and thus an odd number of equilibria. This completes the proof.

A complementary node that is on an $(m, j)$-path with just one initial node will be said to be $(m, j)$-accessible, and any other is one of an $(m, j)$-inaccessible pair. Clearly, the number of $(m, j)$-accessible complementary nodes is odd.

5. A constructive procedure. The foregoing results suggest a procedure for constructing an equilibrium of an $N$-person game; namely, by finding an $\left(m, j_{m}\right)$ accessible complementary node of $Z\left(m, j_{m}\right)$ for an arbitrary player $m \in P$ and some one of his pure strategies $j_{m} \in S_{m}$.

Let $m=N$ and suppose that one has specified $\left(n, j_{n}\right)$ for each $n \in P, n \neq 1$. Then one has a 1-person game $\Gamma_{1}\left(\left\{\left(n, j_{n}\right) \mid n>1\right\}\right)$ played by the first player for which one can readily find the unique equilibrium. This equilibrium provides the unique initial node in $Z\left(2, j_{2}\right)$ for the 2-person game $\Gamma_{2}\left(\left\{\left(n, j_{n}\right) \mid n>2\right\}\right)$ from which one can proceed along the $\left(2, j_{2}\right)$-path containing it to find the unique $\left(2, j_{2}\right)$ accessible complementary node (as described previously by Lemke and Howson [1]). In turn, this node provides an initial node in $Z\left(3, j_{3}\right)$ for the 3 -person game $\Gamma_{3}\left(\left\{\left(n, j_{n}\right) \mid n>3\right\}\right)$. Continuing in this fashion, one wants in general to find a complementary node in $Z\left(k-1, j_{k-1}\right)$ for the game $\Gamma_{k-1}\left(\left\{\left(n, j_{n}\right) \mid n>k-1\right\}\right)$ to provide an initial node in $Z\left(k, j_{k}\right)$ for the $k$-person game $\Gamma_{k}\left(\left\{\left(n, j_{n}\right) \mid n>k\right\}\right)$ from which to proceed along the $\left(k, j_{k}\right)$-path containing it in order to find a $\left(k, j_{k}\right)$ - 
accessible complementary node. When this procedure has been completed for $k=N$ one has found an $\left(N, j_{N}\right)$-accessible complementary node for the original problem and therefore an equilibrium. For $k \geqq 3$, however, the procedure is unlike Lemke and Howson's algorithm in that not every initial node need be on a $\left(k, j_{k}\right)$-path containing a complementary node, and it is the circumvention of this difficulty that we must explain below.

In order to illustrate the basic idea most simply, suppose for the moment that the $(k-1)$-person game has only one $\left(k-1, j_{k-1}\right)$-accessible complementary node, all others being grouped into inaccessible pairs; e.g., this is the case for $k-1=2$ since there is only one initial node. Of course it is precisely this complementary node that will be found by the procedure when one is ready to begin working on the $k$-person game. Now, since one has found the accessible complementary node in the $(k-1)$-person game, it provides an initial node for the $k$-person game. This initial node, say $x$, is either complementary (in which case one is finished if $k=N$ or else one moves on to the $(k+1)$-person game) or there is a unique bounded $\left(k, j_{k}\right)$-almost-complementary arc along which one can move to traverse the $\left(k, j_{k}\right)$-path containing $x$. This path terminates in either a complementary node (in which case again one moves on to the $(k+1)$-person game) or another initial node. In the latter case, observe that this initial node must arise from a complementary node of the $(k-1)$-person game which is one member of a $\left(k-1, j_{k-1}\right)$-inaccessible pair. Hence, from this member there is a unique $\left(k-1, j_{k-1}\right)$-almost-complementary arc which one can traverse in the $(k-1)$-person game which leads to the other member of the pair. This second member provides a new initial node for the $k$-person game, on a new $\left(k, j_{k}\right)$-path, from which one can begin again. Continuing in this way, one must find an initial node that leads to a $\left(k, j_{k}\right)$-accessible complementary node for the $k$-person game. To see this, recall that the number of initial nodes is odd and at least one is on a path containing a complementary node; also, one cannot return to an initial node previously encountered since that would imply, if it were $x$, that the equilibrium from which $x$ arises is $\left(k-1, j_{k-1}\right)$-inaccessible, or if it were any other, that there are three $\left(k-1, j_{k-1}\right)$-inaccessible complementary nodes on a single $\left(k-1, j_{k-1}\right)$-path in the $(k-1)$-person game.

The procedure in the general case is merely a variant of the above. In general, one must allow for the possibility that in the course of the above procedure one finds a new initial node in the $k$-person game that arises from a $\left(k-1, j_{k-1}\right)$ accessible complementary node in the $(k-1)$-person game, rather than one member of an inaccessible pair. In this case, one proceeds along the $\left(k-1, j_{k-1}\right)$ path containing the accessible complementary node in the $(k-1)$-person game to reach its initial node, which arises from a complementary node in the $(k-2)$ person game. If this complementary node is $\left(k-2, j_{k-2}\right)$-accessible in the $(k-2)$-person game, then one repeats. After not more than $k-2$ iterations one must reach a complementary node in a $(k-v)$-person game which is $\left(k-v, j_{k-v}\right)$ inaccessible and therefore is one member of an inaccessible pair, the other member of which provides a new initial node of the $(k-v+1)$-person game from which one can again continue. As before, this procedure cannot cycle.

THEOREM 2. One has a constructive procedure for finding an equilibrium of an $N$-person noncooperative game. 
The term "constructive" as employed here presumes, of course, that one has a means of finding the node at the endpoint of any bounded arc traversed in the course of the procedure. Since for $N \geqq 3$ this requires the solution of a set of simultaneous multilinear equations, at least with a sufficient degree of numerical accuracy, this is by no means a trivial presumption.

Acknowledgment. The author is indebted to Stefan Bloomfield for noticing a deficiency in the original version of this paper.

\section{REFERENCES}

[1] C. E. Lemke AND J. T. Howson, JR., Equilibrium points of bimatrix games, this Journal, 12 (1964), pp. 413-423.

[2] R. Duncan Luce and Howard Raiffa, Games and Decisions, John Wiley, New York, 1957.

[3] JoHN NASH, Non-cooperative games, Ann. of Math., 54 (1951), pp. 286-295. 